Caso clínico

dx.doi.org/10.35366/CMA201K

\title{
Consideraciones anestésicas en el síndrome de Moebius: a propósito de un caso
}

\section{Anesthetic considerations in Moebius syndrome: apropos of a case}

Dra. María Belén Rodríguez-Campoó, ${ }^{*}$ Dr. Pablo Troncoso, ${ }^{\ddagger}$ Dra. Patricia Catalán ${ }^{\ddagger}$

RESUMEN. El síndrome de Moebius es una entidad rara que puede acompañarse de alteraciones que dificulten la anestesia, tales como dificultad de venoclisis, mayor incidencia de depresión respiratoria o de vía aérea difícil. En ocasiones se presenta de forma atípica y puede tener alteraciones del gen RyR con susceptibilidad a hipertermia maligna. Nuestro objetivo en este caso clínico es revisar la preparación necesaria para estos pacientes previa al acto anestésico y quirúrgico, centrándonos en la hipertermia maligna.

ABSTRACT. Moebius syndrome is an uncommon illness that can be associated with different alterations related to anesthesia such as difficult peripheral venous catheterization, more respiratory failure incidence or difficult airway. When this syndrome is accompanied by atypical presentation it can be associated with RyR gen alterations and malignant hyperthermia susceptibility. Our objective with this case is to review which is the preparation required in order to a general anesthesia, focusing on malignant hyperthermia.

Abreviaturas:

${ }^{\circ} \mathrm{C}$ : grados centígrados.

Mg: miligramos.

G: gauge.

Unidades/L: unidades por litro.
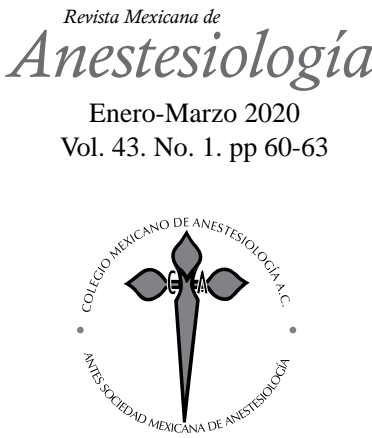

* Departamento de Anestesiología, Hospital Central de la Defensa Gómez Ulla, Madrid.

${ }^{\ddagger}$ Departamento de Anestesiología, Hospital Universitario Niño Jesús, Madrid.

Palabras clave:

Síndrome de Moebius, anestesia general, hipertermia maligna.

Keywords:

Moebius syndrome, general anesthesia, malignant

hyperthermia.

Solicitud de sobretiros:

María Belén Rodríguez-Campoó HCD Gómez Ulla (Madrid)

Tel: +34 669681891

E-mail: belenrcampoo@gmail.com

Recibido para publicación: 30-08-2018

Aceptado para publicación:

09-08-2019

\section{INTRODUCCIÓN}

E l síndrome de Moebius se define como ausencia de expresión facial, estrabismo y dificultades para hablar o tragar. Es secundario a una disrupción en el desarrollo de los pares craneales VI y VII. Como reto para los anestesiólogos, son pacientes que por sus alteraciones de miembros pueden dificultar la venoclisis, pueden presentar vía aérea difícil por una apertura de boca limitada, apneas centrales así como padecer cardiopatías. Todo esto determina la necesidad de una correcta premedicación y buen manejo anestésico perioperatorio ${ }^{(1)}$. En este caso, el niño presenta una mutación RyR:p:His 3981Tyr que puede asociarse con mutación en el gen RYR1 que codifica el receptor de rianodina 1 por lo que es susceptible de hipertermia maligna ${ }^{(2)}$.

Presentamos un caso clínico de un niño con síndrome de Moebius, intervenido de criptorquidia y fimosis, y ex- ponemos las consideraciones anestésicas necesarias dada la mutación RYR que lo hace susceptible de hipertermia maligna.

La alteración del gen RyR aumenta el riesgo de hipertermia maligna (HM). Esta enfermedad tiene una supervivencia altamente dependiente del rápido reconocimiento del cuadro y de su tratamiento. Este paciente presentaba mayor riesgo de padecer HM, motivo por el que repasamos las consideraciones y el manejo de las crisis de HM.

\section{PRESENTACIÓN DEL CASO}

Niño de seis años y 19 kilogramos que es intervenido de criptorquidia y fimosis. Entre sus antecedentes destaca la intervención previa de estrabismo, síndrome de Moebius (de reciente diagnóstico) y trastorno autista. En la consulta preoperatoria no se observaron alteraciones cardiovasculares, debilidad muscular, apneas centrales, insuficiencia respira- 
toria o escoliosis, por lo que es diagnosticado de síndrome de Moebius atípico. Presenta de alteraciones faciales leve retrognatia y limitación de apertura bucal.

Además, presenta una mutación RyR:p:His 3981Tyr con probable miopatía de centrocore o minicore por lo que puede asociar mutación del gen de la rianodina lo que le hace susceptible a hipertermia maligna. También se resalta la relación entre el síndrome de Moebius con el aumento del riesgo de sufrir hipertermia maligna. La analítica no presenta hallazgos relevantes. Estado físico II de la American Society of Anesthesiologist (ASA II).

\section{RESULTADOS}

Se prepara el quirófano para realizar una anestesia libre de desencadenantes de hipertermia maligna. Se reservó un quirófano, se cambiaron las tubuladuras y la cal sodada y se dejó que el respirador ciclara durante más de 100 minutos $^{(3)}$ (Figuras 1 y 2) para conseguir la menor concentración de moléculas de anestésico halogenado (sevoflurano en este caso). Se revisó el contenido y las caducidades del kit de hipertermia maligna, se trajo y se tuvo dentro del quirófano en todo momento (Figura 3). Para intentar minimizar al máximo el riesgo de exposición a triggers se decidió como plan inicial intentar una anestesia regional (intradural) con sedación. Se preparó una anestesia total intravenosa (propofol y remifentanilo) como plan alternativo. La monitorización fue la estándar (electrocardiograma, pulsioximetría y presión arterial no invasiva) incluyendo capnografía y sonda de temperatura (rectal). Descartamos monitorización invasiva porque el niño presentaba un ecocardiograma sin alteraciones, la cirugía era poco invasiva y de corta duración (menor de

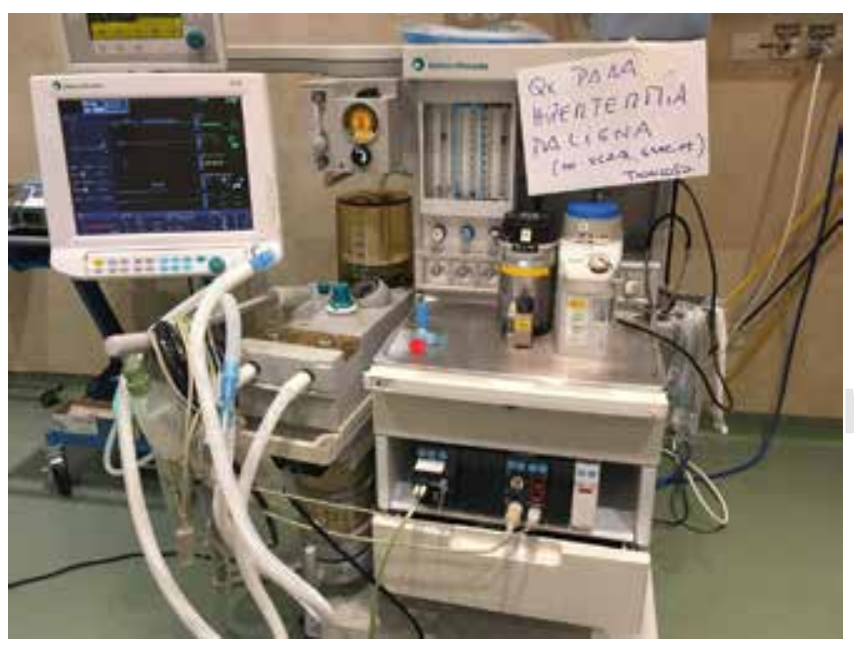

Figura 1: Máquina de anestesia reservada para el caso.

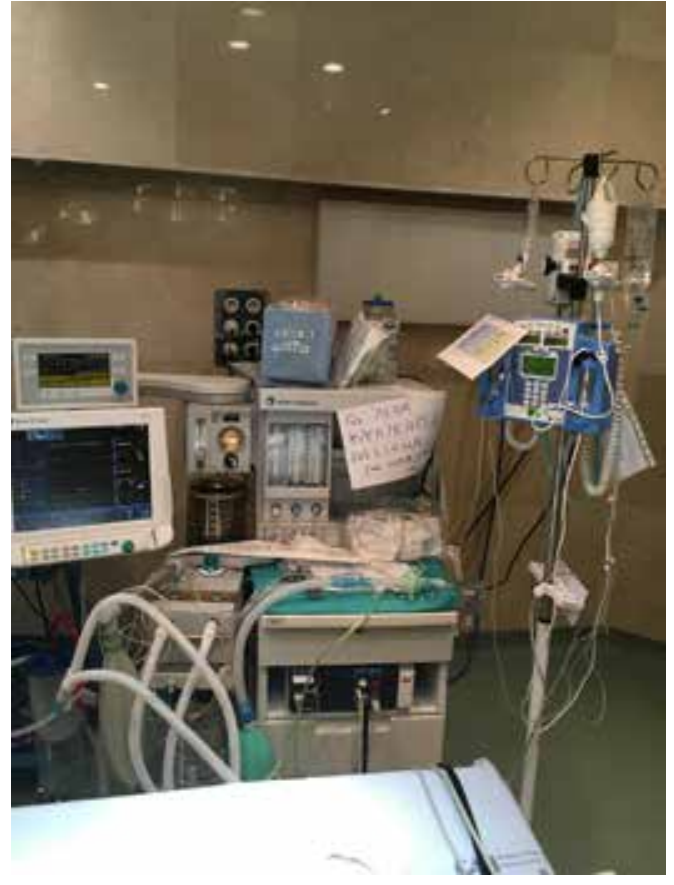

Figura 2: Máquina de anestesia y TIVa preparadas para el caso.

una hora) y por la estabilidad hemodinámica del niño en todo momento. La venoclisis se realizó sin incidencias ni dificultad con anestesia inhalatoria con óxido nítrico. Para la sedación se empleó propofol (10 mg) y ketamina (20 $\mathrm{mg}$ ). La anestesia intradural fue llevada a cabo con técnica aséptica estricta en el espacio L4-L5 con una aguja del 27 G con 7 miligramos de bupivacaína hiperbara $0.5 \%$ y 10 microgramos de fentanilo sin incidentes. Se administró 0.8 gramos de metamizol.

Por otra parte se contemplaron los posibles riesgos del síndrome de Moebius, tales como la prevalencia de vía aérea difícil. No se premedicó por el riesgo que padecen de sufrir apneas centrales.

La intervención duró 45 minutos y fue realizada sin complicaciones. El paciente estuvo estable hemodinámicamente, con temperatura $36 \cdot 7-37^{\circ} \mathrm{C}$. Durante el segundo tercio de la intervención, el paciente tuvo un episodio de hipoventilación (disminución de volumen tidal y frecuencia respiratoria), en el que objetivamos una elevación del $\mathrm{EtCO}_{2}$ hasta $60 \mathrm{mmHg}$ (Figura 4) que revirtió espontáneamente hasta $32 \mathrm{mmHg}$ a los 15 minutos.

Tras ello, el paciente fue ingresado en la Unidad de Reanimación durante 24 horas para observación y prevención de posibles efectos adversos. La recuperación de la anestesia intradural transcurrió sin incidencias. Fue dado de alta de la Unidad de Reanimación y de la estancia en planta hospitalaria sin registrar ningún evento adverso. 


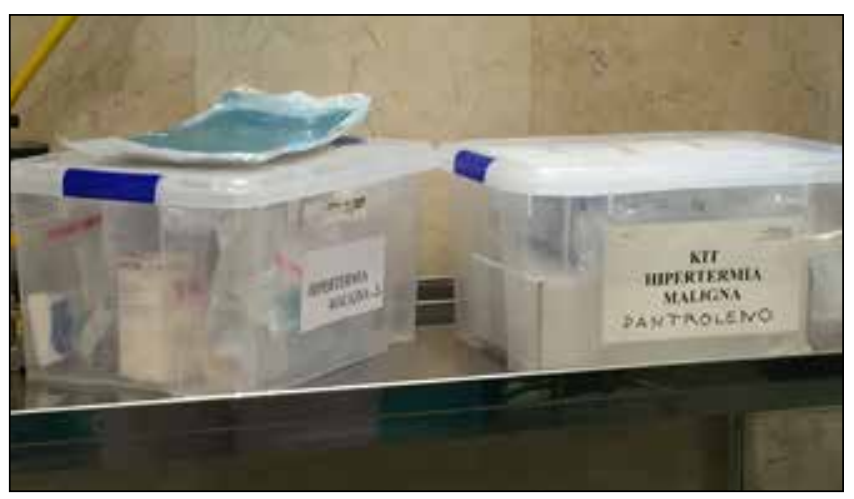

Figura 3: Kit de hipertermia maligna. Se dejó dentro del quirófano tras comprobar que tenía todos los componentes, incluido el dantroleno.

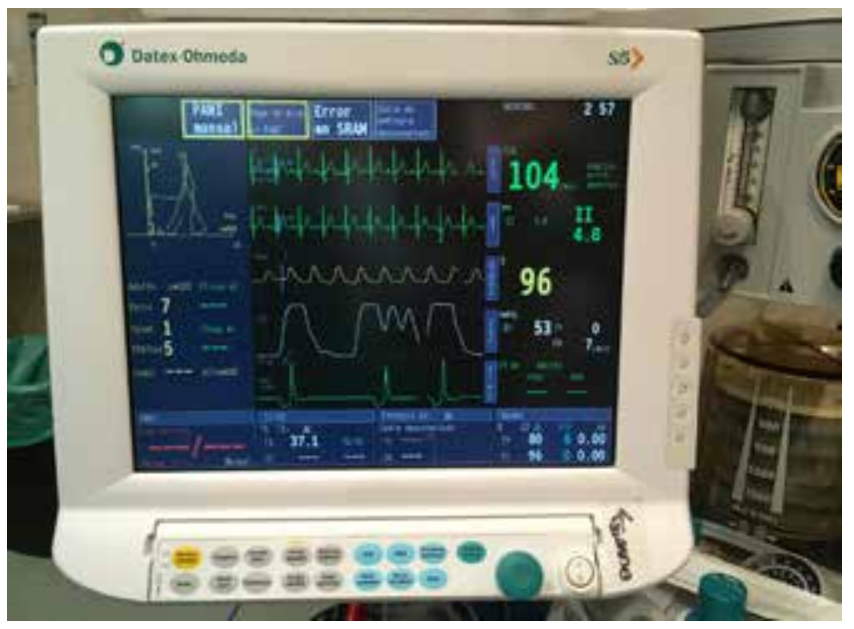

Figura 4: Episodio de hipercapnia asociado a hipoventilación.

\section{DISCUSIÓN}

El síndrome de Moebius fue descrito en 1888 por Paul Julius Moebius. Su etiología no es clara, pero es producto de un desarrollo embrionario anormal ${ }^{(4)}$. La etiología más defendida es la disrupción de la morfogénesis de las arterias basilar o vertebral en un estadío crítico para la gestación. Dicha alteración se traduce en una ausencia de desarrollo de los núcleos de los pares craneales VI y VII y la aparición de calcificaciones y aplanamiento de la base del cuarto ventrículo. Además de la exposición a ciertos medicamentos tales como benzodiacepinas, alcohol, cocaína y el recientemente relacionado misoprostol ${ }^{(1,4)}$. La mayoría de los pacientes no presentan relación genética, pero se ha documentado casos con transmisión autosómica. Los dos locus implicados se localizan en los cromosomas 3 y 13 (3q21-q22 y 13q12.1-q13, respectivamente) ${ }^{(5,6)}$.

Las manifestaciones clínicas son: baja implantación del pabellón auricular, anomalías de los oídos, facies inexpresiva, micrognatia, microstomía, apertura oral limitada y sobrecrecimiento premaxilar. También se ha encontrado relación con casos de labio leporino y paladar hendido y se ha asociado a ausencia de pectoral en el síndrome de Moebius-Poland).

La afectación de VI y VII pares craneales son los responsables del cierre incompleto de los párpados durante el sueño, úlceras corneales, escurrimiento de saliva, estrabismo y ausencia de movimientos de expresión facial ${ }^{(7)}$. No es infrecuente encontrar otros pares craneales afectos tales como V (trigémino), IX (glosofaríngeo), X (vago) y XII (hipogloso) ${ }^{(7)}$. Se puede asociar a trismus, hipoacusia, hipotonía muscular e incluso disfunción respiratoria central. En 50\% de los casos se ha encontrado asociación a anormalidades de las extremidades, tales como pie equino, sindactilia, amputación congénita, etcétera. También puede haber algún grado de retardo mental ${ }^{(5)}$ y alteraciones cardiovasculares ${ }^{(8)}$.

Los pacientes con síndrome de Moebius precisan de intervenciones quirúrgicas para corrección de algunas de las anormalidades que se describen. La cirugía más frecuente ${ }^{(4)}$ es el trasplante segmentario del músculo gracilis para mejorar la expresión facial y evitar la salida involuntaria de la saliva.

Las implicaciones anestésicas fueron estudiadas por primera vez por el grupo formado por Ames y colaborador ${ }^{(4)}$, estudiando sobre todo la dificultad en la intubación traqueal. Como otras enfermedades neuromusculares, hay que tener en cuenta que pueden aparecer cinco complicaciones frecuentes ${ }^{(9)}$ : miotonía que va acompañada de liberación de potasio, proteínas musculares y mioglobinuria, rabdomiólisis, hipertermia maligna así como parálisis prolongada. La canalización vascular de estos pacientes puede ser un reto, debido a la alteración frecuente de las extremidades. La evaluación preanestésica es fundamental. La presencia de cardiopatía en $21 \%$ de los sujetos obliga a considerar evaluación cardiológica pertinente y profilaxis antibiótica si precisa ${ }^{(8)}$. La valoración de la vía aérea debe de ser rigurosa y considerarse de antemano una vía aérea difícil. En nuestro caso se observó dificultad de apertura bucal y Mallampati III, por dicho motivo se dejó el fibroscopio dentro del quirófano así como diferentes dispositivos y una mascarilla laríngea por si la intubación orotraqueal ${ }^{(10)}$ fuese necesaria y el paciente presentara una vía aérea difícil. Se debe intentar conservar la ventilación espontánea y evitar el uso de relajantes musculares.

La inducción anestésica debe ser cuidadosa y considerar la posibilidad de que el sujeto tenga apnea central. Se deben administrar opioides y benzodiazepinas con cautela ${ }^{(5)}$. El uso de succinilcolina debe evitarse al ser un trigger de la hipertermia maligna, haciendo hincapié en el elevado riesgo de desarrollar fallo respiratorio.

En el intraoperatorio hay que estar atento a las posibles complicaciones que pueden presentarse. El riesgo para desarrollar úlceras corneales aumenta, por ello es necesario el empleo de adhesivos y lubricantes. Se recomienda evitar el 
uso de anestésico inhalatorios en aquéllos con síndrome de Moebius atípico con alteración del gen RyR por el aumento de riesgo de hipertermia maligna y de depresión miocárdica ${ }^{(2,7)}$.

La anestesia regional no está contraindicada, aunque debemos tener en cuenta que $5 \%$ de los pacientes pueden tener neuropatía periférica previa ${ }^{(8)}$.

La hipertermia maligna (HM) es un trastorno farmacogenético de la musculatura esquelética que se caracteriza por un estado hipermetabólico tras una anestesia con succinilcolina y/o agentes halogenado. Existe relación entre varios síndromes neuromusculares y el aumento del riesgo de sufrir HM. El dantroleno es el fármaco de elección para el tratamiento.

Los criterios diagnóstico para la HM son acidosis respiratoria por $\mathrm{CO} 2>55 \mathrm{mmHg}, \mathrm{PaCO}_{2}$ en gasometría arterial > 60 mmHg, manifestación cardíaca inexplicada caracterizada por taquicardia sinusal, taquicardia ventricular o fibrilación ventricular, acidosis metabólica demostrada por déficit de base $>8 \mathrm{mEq} / \mathrm{L}, \mathrm{pH}>7.25$, rigidez muscular generalizada, importante rigidez del músculo masetero, CPK > 20,000 unidades/L, orina oscura, potasio sérico $>6 \mathrm{mEq} / \mathrm{L}$, elevación rápida de temperatura por encima de $38.8^{\circ} \mathrm{C}$, historia familiar (herencia autosómica dominante) y reversión rápida de los signos de HM tras la administración de dantroleno ${ }^{(11,12)}$. Nuestro paciente no presentó ningún signo de los anteriores y el aumento de $\mathrm{EtcO}_{2}$ coincidió con un episodio de hipoventilación.
Es esencial la identificación y el manejo precoz de la crisis aguda de HM, por ello consideramos oportuno tener el kit de hipertermia maligna dentro del quirófano. El tratamiento se basa en la interrupción de la administración del fármaco desencadenante, el dantroleno (primero en bolo $2.5 \mathrm{mg} / \mathrm{kg}$ y después en infusión) y en medidas de soporte (hiperventilar, medidas de enfriamiento, tratamiento de arritmias) ${ }^{(12)}$. Se recomienda derivar al paciente y familiares a un centro que realice el test de contractura de cafeína-halotano o test de ADN.

La prevención en los casos de riesgo debe ser siempre considerada. Se debe priorizar evitar los fármacos desencadenantes (anestésicos halogenados y relajantes neuromusculares). Para evitar que ninguna partícula de gas halogenado pase al paciente en riesgo de sufrir una $\mathrm{HM}$, es preciso dejar ciclando la máquina de anestesia un tiempo que depende del tipo de máquina ${ }^{(13)}$, nosotros empleamos una Datex-Ohmeda Aestiva, motivo por el cual dejamos ciclando la máquina a altos flujos más de 90 minutos $^{(13)}$.

\section{CONCLUSIONES}

El síndrome de Moebius es una entidad rara y heterogénea. Debemos conocer el espectro clínico que abarca, así como el riesgo aumentado a diferentes eventos adversos durante un proceso anestésico. En algunos casos de síndrome de Moebius atípico, se puede encontrar alteración del gen de la rianodina, por lo que se deben extremar las precauciones para evitar un episodio de hipertermia maligna.

\section{REFERENCIAS}

1. Cavieres MF. Developmental toxicity of misoprostol: an update. Rev Med Chile. 2011;139:516-523.

2. Shaaban S, Ramos-Platt L, Gilles FH, Chan WM, Andrews C, De Girolami U, et al. RYR1 mutations as a cause of ophthalmoplegia, facial weakness, and malignant hyperthermia. JAMA Ophthalmol. 2013;131:1532-1540.

3. Glahn KPE, Ellis FR, Halsall PJ, et al. Recognizing and managing a malignant hyperthermia crisis: guidelines from the European Malignant Hyperthermia Group. Br J Anaesth. 2010;105:417-420.

4. Ames WA, Shichor TM, Speakman M, Zuker RM, McCaul C. Anesthetic management of children with Moebius sequence. Can J Anaesth. 2005;52:832-844.

5. Gondipalli P, Tobias JD. Anesthetic implications of Möbius syndrome. J Cin Anesth. 2006;18:55-59.

6. Uzumcu A, Karaman B, Toksoy G, Uyguner ZO, Candan S, Eris H, et al. Molecular genetic screening of MBS1 locus on chromosome 13 for microdeletions and exclusion of FGF9, GSH1, CDX2 as causative genes in patients with Moebius syndrome. Eur J Med Genet. 2009;52:315-320.
7. Poveda R. Secuencia de Moebius en Anestesiología. Rev Chil Anest. 2012;41:140-143.

8. Ferguson S. Moebius syndrome: a review of the anesthetic implications. Paediatr Anaesth. 2006;6:51-56.

9. Forés B, Manzano A, Urrengoetxea P, Aguilera L. Anestesia en el paciente con patología neuromuscular. Gac Med Bilbao. 2006;103:79-89.

10. Hobaika AB, Neves BS, Fernandes ML, Guedes VC. Anesthesia in a patient with Moebius sequence: case report. Rev Bras Anestesiol. 2009;59:341-343.

11. Krajcirik WJ, Azar I, Opperman S, Lear E. Anesthetic management of a patient with Moebius syndrome. Anesth Analg. 1985;64:371-372.

12. Rosenberg H, Davis M, James D, Pollock N, Stowell K. Malignant hyperthermia. Orphanet J Rare Dis. 2007;24:21.

13. Jones C, Bennett K, Kim TW, Bulger TF, Pollock N. Preparation of Datex-Ohmeda Aestiva and Aisys for anaesthetic machines for use in malignant hyperthermia susceptible patients. Anaesth Intensive Care. 2012;40:490-497. 